\title{
Anomalous Shape and Decay of Islands on Au(110)
}

\author{
M. J. Rost, R. van Gastel, and J. W. M. Frenken \\ Kamerlingh Onnes Laboratory, Leiden University, P.O. Box 9504, 2300 RA Leiden, The Netherlands
}

(Received 13 October 1999)

\begin{abstract}
Scanning tunneling microscopy on roughened $\mathrm{Au}(110)$ reveals that the equilibrium shape of islands and pits on this surface is almondlike: each island contains two smoothly curved steps joined at two sharp corners. This shape has recently been predicted and finds its origin in the missing-row reconstruction of its fcc (110) surfaces (Au, Pt, etc.). We use the corner angles and the island shapes to determine the step energies. In addition we find that during the decay of an island on the $\mathrm{Au}(110)$ surface the shape changes and that the disappearance of the island involves the splitting of the layer below the island into two disconnected regions. The shape change has a dramatic influence on the decay rate of the islands.
\end{abstract}

PACS numbers: 68.35.Md, 61.16.Ch, 68.35.Bs, 82.65.Dp

The equilibrium shape of a crystal can be found via the well-known Wulff construction from the orientationdependent surface free energy [1]. Equivalently, one can obtain the two-dimensional equilibrium shape of an adatom or vacancy island on a flat surface from the step free energies. Usually, such a two-dimensional equilibrium shape contains neither straight sections nor sharp corners at any nonzero temperature. When surface diffusion makes an island disappear, it usually keeps the same equilibrium shape and simply decreases in area.

In this Letter we present a scanning tunneling microscopy (STM) study of island shapes on the Au(110) surface in ultrahigh vacuum. Our observations confirm the recent prediction of a peculiar island shape on this surface, which features two sharp corners [2]. We show that the missing-row reconstruction (MRR), which is responsible for this shape, also modifies the decay of the islands, making the layer below a disappearing island break up into two separate regions. Finally, we show that the islands go through a dramatic change in shape during their decay and that this is reflected in the decay dynamics of the islands.

The measurements have been performed in ultrahigh vacuum with a homebuilt scanning tunneling microscope [3]. The Au sample was mechanically polished in the (110) orientation to within $0.1^{\circ}[4]$ and cleaned in situ by cycles of $\mathrm{Ar}^{+}$ion sputtering (15 ML) at room temperature and annealing to $500{ }^{\circ} \mathrm{C}$. This produced a flat $\mathrm{Au}(110)$ surface with an average terrace width up to $3000 \AA$ along the [001] and up to $10000 \AA$ along the [1110] direction. Mounds and pits were created on this surface by several cycles of sputtering ( $5 \mathrm{ML}$ ) at room temperature and subsequent annealing to $250{ }^{\circ} \mathrm{C}$. At this low annealing temperature the surface had sufficient mobility for the mounds and pits to reach their equilibrium shape but insufficient mobility for these structures to completely decay and restore the flat surface. All STM images were acquired at a sample voltage of $-0.7 \mathrm{~V}$ and at tunneling currents below $0.1 \mathrm{nA}$. Under these tunneling conditions no influence of the $\mathrm{W}$ tip on the Au surface was observed. The scale of the images shown in this Letter are too large to resolve the missing row (MR) structure, but in each case the tip was sufficiently good to resolve a well-defined defect-free MRR everywhere on the surface. In Fig. 1 several mountains and valleys can be seen, each of them several tens of monolayers high and deep. All islands have an almondlike shape: curved sides joined at sharp corners. The right panel in Fig. 1 is an enlarged view of the corners at one side of a mound. Two types of corners can be found: (i) corners where the steps seem to cross; we will further refer to such corners as crossing sites; (ii) locations where two pairs of steps reach the corner from the same side and end there; we will call these termination sites. We find that on a misoriented (part of the) $\mathrm{Au}(110)$ surface the steps are organized in a fish-scale-type network with crossing sites [5]. Termination sites are used to accommodate curvature of the surface. As explained in the following, the origin of the almond shape, the corner configurations, and the network structures lie in the $(1 \times 2)$ missing-row reconstruction of $\mathrm{Au}(110)$. Figure 2(a) shows a cross section of the reconstructed Au surface. Every second surface atom row along the [110] direction is missing. Figures 2(b)-2(d) illustrate three potential island structures on this surface. The simplest way to create an island

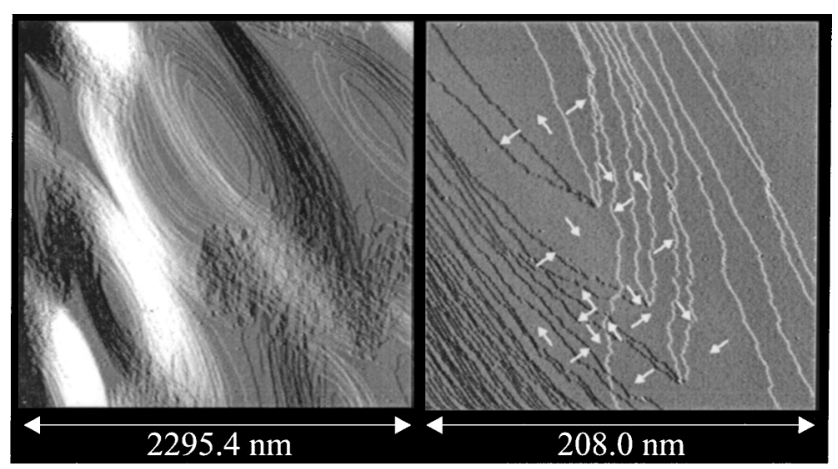

FIG. 1. STM images (differentiated) of the artificially roughened surface. Mounds and pits have almondlike shapes. The right panel illustrates the two possible corner configurations: crossing sites and termination sites. Arrows denote the phase (see text) of the MRR. 


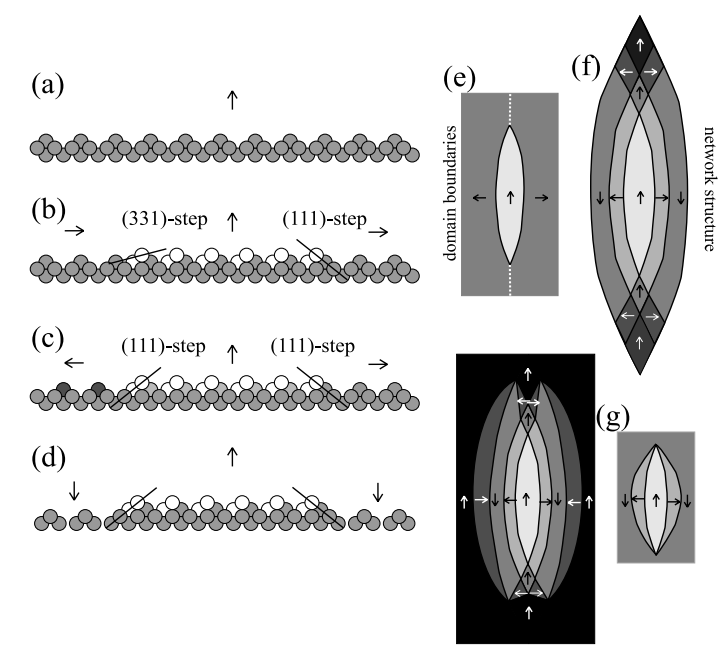

FIG. 2. Schematic top views and cross sections perpendicular to the MRR. (a) Terrace. (b) Adatom island with different steps. (c) Adatom island with (111) steps and a phase difference between left and right. (d) Double-height island. (e) Single adatom island with domain boundary in the layer below (dotted line). (f) Multilevel island without domain boundaries. (g) Alternative multilevel islands without domain boundaries. Note that the steps always meet each other in groups of four.

would be to place extra Au atoms directly on the MR reconstructed surface, but this would result in nonequivalent steps on the two sides of the island, which we denote as (111) and (331) steps to indicate the orientation of the corresponding nanofacet [Fig. 2(b)]. Unless the surface is strongly pinned by defects, we never observe (331) steps. Their formation energy $\delta_{[1 \overline{1} 0]}^{331}$ must be so much higher than that of the (111) steps, $\delta_{[110]}^{111}$, that the surface resorts to an alternative island structure. There are several possibilities for the surface to accommodate islands with only (111)-type edges. One is to introduce a phase shift in the reconstruction of the substrate. At each (111) step the missing-row pattern shifts by one-quarter of the regular missing-row spacing. We indicate the four phases [6] with arrows; see, e.g., parts $\leftarrow$ and $\rightarrow$ in Fig. 2(c). Crossing a (111) step to the (left) right we rotate the arrow $90^{\circ}$ (counter-) clockwise. Another possibility is to make all mounds and pits an even number of monolayers high or deep [Fig. 2(d)]. We observe that $\mathrm{Au}(110)$ invariably chooses single-height island configurations with a phase shift [Fig. 2(c)]. One might expect that this would unavoidably lead to situations as sketched in Fig. 2(e), with domain boundaries between the phase shifted parts of the substrate. However, such configurations are not observed at room temperature. Instead, the steps always meet in crossing or termination sites [Figs. 2(f) and $2(\mathrm{~g})]$, and in this way effectively reduce all domain boundary lengths to zero. The high formation energy of the (331) step has a major effect on the free energy of a step oriented along the [001] direction, i.e., perpendicular to the MR direction. Such a step has a high free energy since it has great difficulty meandering. This is because the meandering would introduce both (111) and (331) regions along the step, and the latter are high in energy. Under the assumption that (331) steps cannot be formed, so that steps along [001] can be only straight, Carlon and van Beijeren have computed the orientation-dependent free energy of steps on $\mathrm{Au}(110)$ [2]. They find that the steps indeed exhibit a distinct maximum in the free energy along [001] and that this orientation plus a (temperature dependent) range of step orientations around it is expelled from the equilibrium island shape. As a consequence the equilibrium island shape looks like an almond: each island contains two sharp corners where two (111)-type steps (with kinks) are joined under a well-defined angle $2 \phi$ (see Fig. 3). This angle is determined by $\delta_{[1 \overline{1} 0]}^{111}$, by the energy $\delta_{[001]}$ of the step along [001], and by temperature. We have measured the angles $\phi$ for mounds and pits formed at temperatures ranging from 300 to $394 \mathrm{~K}$ and found values scattered between $8^{\circ}$ and $32^{\circ}$. As we explain below, this spread in $\phi$ does not come from measurement errors or random statistical variations in the island shape. Instead, it reflects the fact that most islands do not exhibit one unique, size-independent equilibrium shape.

We first continue by investigating the smoothening of mounds and pits on $\mathrm{Au}(110)$ at elevated temperatures. Figure 4 shows four stages in the evolution of the topmost two atomic levels of a mound at $394 \mathrm{~K}$. Between the third and the fourth images the topmost terrace disappears completely. During its disappearance, the layer below splits into two parts. We observe this unusual behavior for every terrace that disappears on $\mathrm{Au}(110)$. The explanation again lies in the MRR. As illustrated by Figs. 2(c) and 2(f), the MRR in the layer below the disappearing island carries a phase difference between the regions left and right of the island. When the upper terrace is removed, either a domain boundary has to be left behind or the newly exposed layer has to break up into two separate regions, thereby forming two new (111) steps. The surface avoids the formation of domain boundaries, and chooses the second possibility, which is to form new steps. This is consistent with the observed mound and pit configurations with crossing and termination sites, discussed above (Fig. 1). The preference for steps over domain boundaries implies that the energies of steps $\delta_{[1 \overline{1} 0]}^{111}$ and domain boundaries $\delta_{\mathrm{DB}}$ satisfy the following inequality: $2 \delta_{[1 \overline{1} 0]}^{111}<\delta_{\mathrm{DB}}$. Figure 5

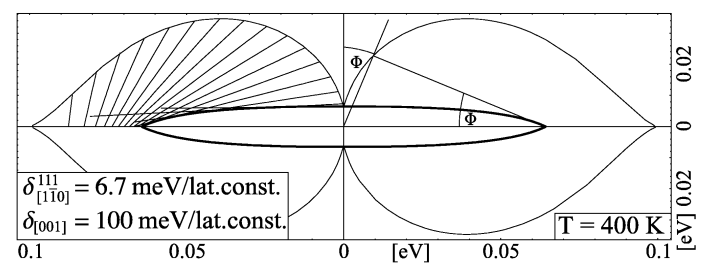

FIG. 3. Two-dimensional Wulff construction of an adatom or vacancy island (thick line). The thin line is the step free energy. Steps with an angle larger than $22.6^{\circ}$ are expelled from the equilibrium shape (see Ref. [2]). 


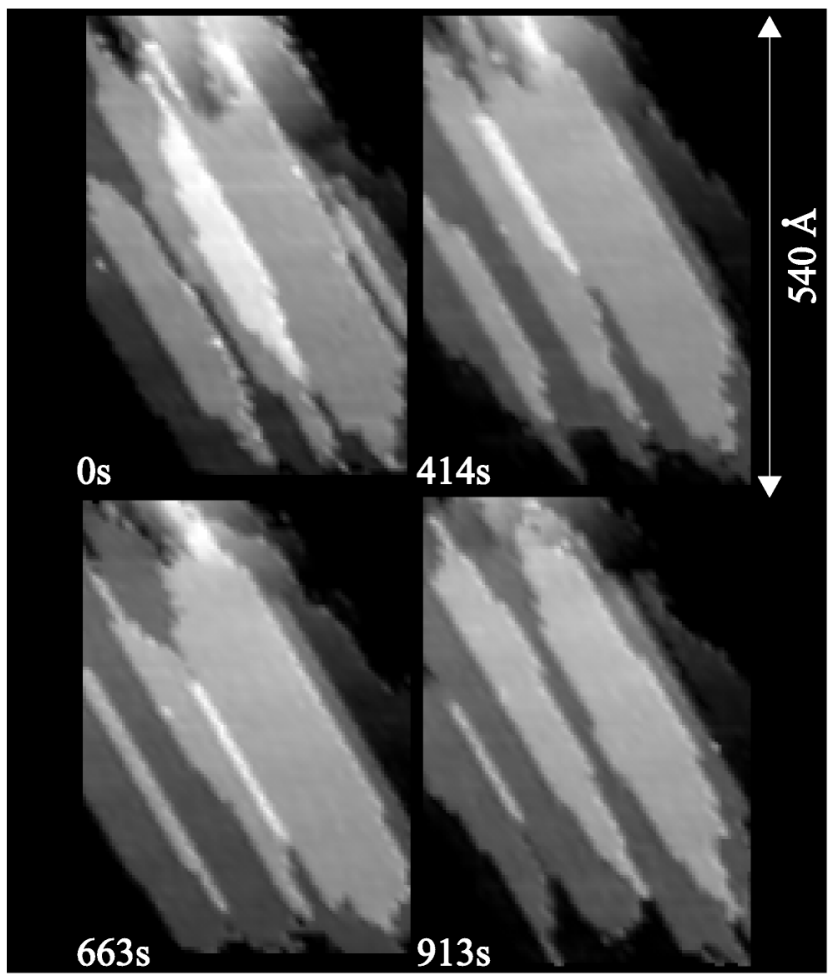

FIG. 4. STM images of the disappearance of the topmost level of a mound at $394 \mathrm{~K}$. The island becomes very narrow during its final stages. The layer below splits into two parts.

schematically illustrates the decay process of mounds (and pits) on $\mathrm{Au}(110)$. The crossing site network allows us to describe each step configuration as a set of extended, curved lines that cross at the crossing sites. The removal of the topmost terrace can then be regarded as the result of a decrease in curvature and/or an outward motion of the two steps that define that terrace. At first sight it might seem that the sequence of subsequent splitting of lower and lower levels would keep increasing the density of terraces. But, as the numbers in Fig. 5 indicate, the total number of terraces is actually reduced by two for every island that disappears.

The second observation that we make in Fig. 4 is that the topmost island undergoes a dramatic change in shape during its evolution. The island on the left in the second layer in Fig. 4 exhibits a similar shape change. First the islands become increasingly narrow, and finally they lose their almond shape completely, forming a narrow strip of two to three close-packed atom rows, which reduces in length until the island is completely removed. Again, this behavior was observed for all disappearing islands on $\mathrm{Au}(110)$. We quantify the shape change by measuring the island's aspect ratio, length/width. The length is measured as the distance along the [1ㅣㅣ direction between the island's crossing sites, and the width is measured along the perpendicular [001] direction, midway between the crossing sites. Figure 6 shows the typical evolution of the area and the aspect ratio of a terrace, from its formation to its disappearance.
While the island reduces in size, the aspect ratio increases from an initial value of 5.5 to a maximum value of 19 at $6200 \mathrm{~s}$. The wiggles result from the loss of individual atom rows. Above $6200 \mathrm{~s}$ the aspect ratio quickly drops and the island disappears. Between 5.5 and 19 the island passes its own equilibrium aspect ratio (see below). This means that most of the islands that we observed cannot be considered to have true equilibrium shape, characteristic for an isolated, infinitely large island in equilibrium with a reservoir of $\mathrm{Au}$ adatoms. This explains the large variation in measured corner angles $2 \phi$ and renders the analysis of these angles in terms of step free energies impossible. We speculate that the existence of the network of steps surrounding the topmost terrace invalidates the simple, single-layer interpretation of the shape of that terrace. If we regard the mound configuration as a network of curved lines that cross without slope discontinuities in the crossing points, then the maximum angle at which each line is allowed to run, anywhere on the mound, should be equal to $\phi$. As is clear from Figs. 1 and 2, this maximum angle should occur at the base of the mound, rather than at its top or in the intermediate network [7].

Because a termination site has the possibility to move in all directions, it forms the only structure that can easily adjust itself in order to minimize the free energy of the joining steps. We have measured the average angle at termination sites between 345 and $470 \mathrm{~K}$. Below $345 \mathrm{~K}$, the mobility of the surface was too low to allow equilibration of the angle within half a day. Above $470 \mathrm{~K}$, the surface mobility was too high to allow reliable STM measurements of the step angles. The measured angles $(\phi)$ are shown in Fig. 7. Applying the theory of Ref. [2] we

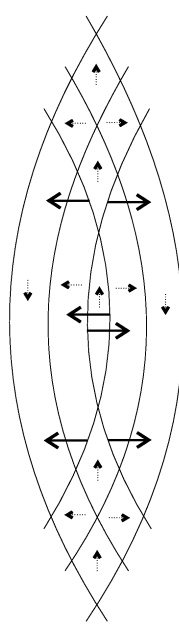

13

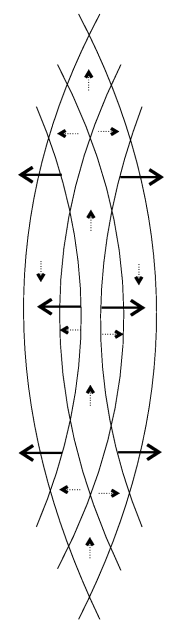

11

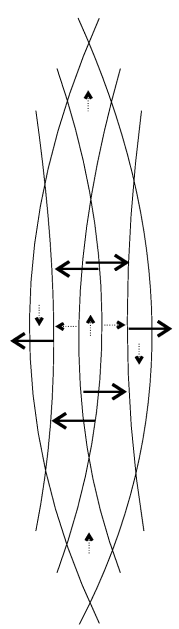

7
FIG. 5. Schematic decay of a mound. The steps recede in the directions indicated by the larger arrows and make the topmost terrace shrink (left). When the topmost terrace is reduced to zero size, the terrace below is divided in two separate parts (middle). The process repeats itself (right). The number of terraces is indicated below each configuration. 


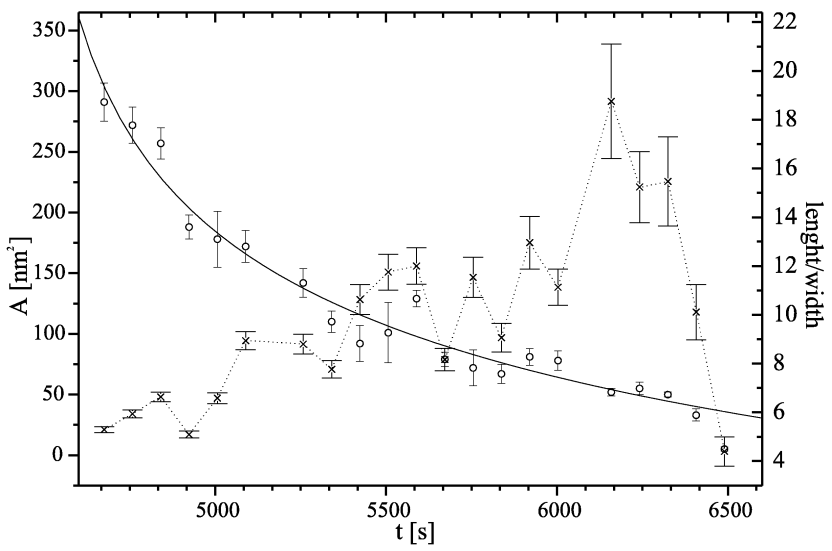

FIG. 6. Measured evolution of the upper terrace of a mound. The terrace area (left axis) is indicated by circles, and the aspect ratio (right axis) by crosses. The solid curve is a fit with a power of $-1 / 5$.

fit these measurements, using $\delta_{[110]}^{111}$ and $\delta_{[001]}$ as fitting parameters. We obtain $\delta_{[1 \overline{1} 0]}^{111}=6.7 \pm 0.7 \mathrm{meV}$ per lattice constant for almost all choices of $\delta_{[001]}$. The step energies are expressed per lattice constant $(4.08 \AA)$. Using the result of Ref. [8] for $\delta_{[1 \overline{1} 0]}^{111}+\delta_{[1 \overline{1} 0]}^{331}=26.9 \mathrm{meV}$ per lattice constant we find $\delta_{[1 \overline{0} 0]}^{331}=20.2 \mathrm{meV}$ per lattice constant, which explains the avoidance of (331) steps of the surface. From the slope $d \phi / d T$ we find $\delta_{[001]} \geq 70 \mathrm{meV}$ per lattice constant. Some top-level islands have approximately the correct angle $\phi$. Comparing the average aspect ratio of these of $8.5 \pm 1.5$ with the Wulff construction, we obtain an upper estimate for $\delta_{[001]}$ of $130 \mathrm{meV}$ per lattice constant. We combine these into $\delta_{[001]}=100 \pm 30 \mathrm{meV}$ per lattice constant.

Finally, we concentrate on the consequences that the shape change of the islands has for the time dependence of their decay. We see in Fig. 6 that the island area $A$ decays more slowly than linearly in time. Once the island has passed the maximum aspect ratio, the decay rate speeds up. This behavior is very different from the $\left(t_{1}-t\right)^{2 / 3}$ behavior that is usually observed for decaying islands [9] and opposite to the anisotropic island decay on $\operatorname{Ag}(110)$ [10]. Since the length change of the islands is always much smaller than their width change (see Fig. 4), we model the decay as follows. We describe the island as the area enclosed between two intersecting circles (both with radius $r$ ) with a fixed distance between the intersection points. The decay is then described by an increase of the radius, implying a decrease of the curvature, a corresponding lowering of the adatom equilibrium pressure, and therefore a slowing down of the decay rate. Under these conditions, $A \sim r^{-1}$, and we obtain $r \sim t^{1 / 5}$, so that $A \sim t^{-1 / 5}$. A fit

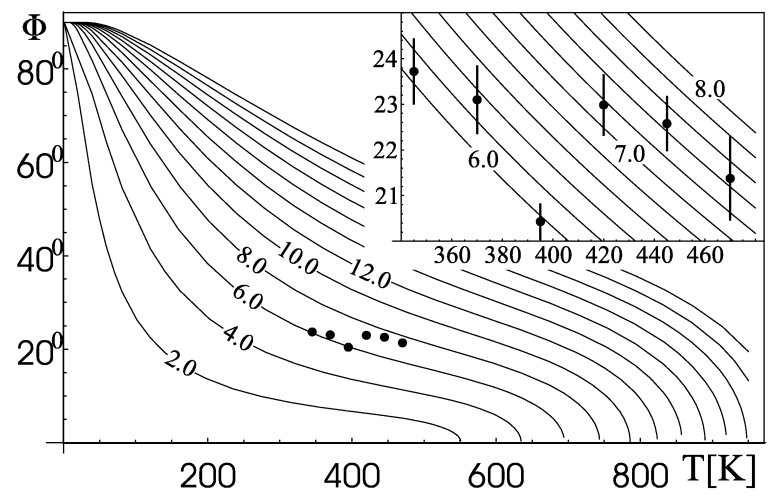

FIG. 7. Angles $\phi$ of termination sites (circles). Curves are calculated (according to Ref. [2]) for the best-fit value $\delta_{[100]}=$ $100 \mathrm{meV}$ per lattice constant and different values for $\delta_{[1 \overline{1} 0]}^{111}$.

with this power law to the first part of the decay is shown in Fig. 6. When the aspect ratio decreases, the island length can no longer be treated as constant. The step curvature quickly increases, and the decay rate should increase dramatically. This is indeed what is observed in Fig. 6.

We expect that most of the phenomena reported here are not exclusive to $\mathrm{Au}(110)$, but that they also occur on (110) surfaces of other fcc materials that exhibit a missing-row reconstruction. Examples of these are clean $\operatorname{Pt}(110), \operatorname{Ir}(110)$, and $\operatorname{Pd}(110)$.

This work is financially supported by the "Nederlandse Organisatie voor Wetenschappelijk Onderzoek (NWO)."

[1] See, e.g., C. Herring, in Structure and Properties of Solid Surfaces, edited by R. Gomer and C. S. Smith (University of Chicago Press, Chicago, 1953), p. 5; I. V. Markov, Crystal Growth for Beginners (World Scientific, Singapore, 1995), pp. 1-41.

[2] E. Carlon and H. van Beijeren, Phys. Rev. Lett. 76, 4191 (1996).

[3] M. S. Hoogeman et al., Rev. Sci. Instrum. 69, 2072 (1998).

[4] R. J. I. M. Koper, www.surface-prep-lab.com

[5] J. K. Gimzewski et al., Phys. Rev. B 45, 6844 (1992).

[6] M. den Nijs, Phys. Rev. Lett. 66, 907 (1991).

[7] K. Swamy, E. Bertel, and I. Vilfan, Surf. Sci. 425, L396 (1999).

[8] M. S. Hoogeman, Ph.D. thesis, Leiden University, 1998.

[9] G. Rosenfeld, K. Morgenstern, and G. Comsa, in Surface Diffusion: Atomistic and Collective Processes, edited by M.C. Tringides (Plenum Press, New York, 1997), p. 361; M.Z. Allmang, L.C. Feldman, and M.H. Grabow, Surf. Sci. Rep. 16, 377 (1992).

[10] K. Morgenstern et al., Phys. Rev. Lett. 83, 1613 (1999). 